Relations industrielles

Industrial Relations

\title{
Index alphabétique : Les relations du travail au Canada Subject Index : Canadian Industrial Relations
}

Volume 25, numéro 1, 1970

URI : https://id.erudit.org/iderudit/028105ar

DOI : https://doi.org/10.7202/028105ar

Aller au sommaire du numéro

Éditeur(s)

Département des relations industrielles de l'Université Laval

ISSN

0034-379X (imprimé)

1703-8138 (numérique)

Découvrir la revue

Citer ce document

(1970). Index alphabétique : Les relations du travail au Canada. Relations industrielles / Industrial Relations, 25(1), 113-126.

https://doi.org/10.7202/028105ar

Tous droits réservés (C) Département des relations industrielles de l'Université Laval, 1970
Ce document est protégé par la loi sur le droit d'auteur. L'utilisation des services d'Érudit (y compris la reproduction) est assujettie à sa politique d'utilisation que vous pouvez consulter en ligne.

https://apropos.erudit.org/fr/usagers/politique-dutilisation/ 


\title{
INDEX ALPHABÉTIQUE
}

\author{
Les Relations dU TRAVAIL aU CaNada \\ ÉQUIPE SPÉCIALISÉE EN RELATIONS DU TRAVAIL (E.S.R.T.)
}

\section{Accréditation}

$61 ; 63 ; 64 ; 259-265 ; 394$.

Accréditation temporaire (construction), 459.

Durée, 464.

Reconnaissance volontaire, 454.

Révocation et cessation, 463; 465; 466.

Syndicats, 454-459.

Vote de représentation, 454.

Acte de l'Amérique du nord britannique 47; 52-56; 752-769.

Action collective 433-446; 530-534.

Action politique syndicale 88-89; 513-518.

Administration du personnel

Définition, 104.

Affiliation syndicale

Changement, 458; 465.

Agents publics

Droit d'association, 439-440.

\section{Aliénation}

Causes, 302-303; 554.

Des travailleurs, 301-306.

Négociation collective, 306.

Syndicats, 304-305.

\section{Arbitrage} 69.

Arbitrage obligatoire, 394-498.

Sentence exécutoire, 603.

Association canadienne de la construction $91 ; 531$.

Association canadienne des manufacturiers 91; 272.

Association canadienne des producteurs de pâtes et papiers 91.

Association d'employeurs 62; 91 .

Accréditation, 531.

Liberté d'association et action collective, 530-534.

Négociation collective, 350 .

Promotion, 535.
Association des chemins de fer du Canada

91.

Associations reconnues

454-455.

Assurance-chômage

56.

Assurance-grève 532-534.

Atelier fermé 324.

Définition, 320.

Atelier syndical

Définition, 320.

Atelier syndical ouvert

Définition, 320.

Australie

Nombre de grèves, 407.

Bilinguisme-biculturalisme

Syndicalisme, 343.

Bonne foi

Négociation collective, 544-548.

Boycottage

611-645.

But du, 620 .

Juridiction fédérale-provinciale, 762-764.

Bureau de placement (voir placement)

Bureau de recherche sur les revenus et les coûts

Coopération fédérale-provinciale, 767.

Fonction, 666-672.

\section{Cadres}

Droit d'association et de négociation, 437.

Canada français 45.

Carter (Commission)

160.

Capitalisme

$30 ; 32 ; 273$.

Centres de décision

Négociation collective, 553-555.

* Cet index alphabétique a été préparé par Jean Sexton afin de faciliter la consultation du rapport Woods. Les numéros qui apparaissent réfèrent aux paragraphes. 


\section{Centralisation}

De la négociation collective, 553-555.

Centre des dirigeants d'entreprise 91.

Chambre de commerce du Canada $91 ; 271 ; 279$.

Changements technologiques (voir conversion industrielle) 288: 673-685.

Et mécontentement des travailleurs, 310.

Charte des Nations Unies 26.

Chômage

Assurance, 56.

Coût du, 162.

Cyclique, 692-694.

Inflation, 652.

Taux de, 159.

Code canadien du travail (normes) 713.

\section{Code criminel} $49 ; 256 ; 442$.

Activité syndicale, 631.

Bris de contrat, 632.

Cerner et surveiller, 633.

Pratiques déloyales, 634.

Restreindre le commerce, 635.

Commission canadienne des relations du travail 734-751.

Commission d'enquête industrielle 574.

Commission de conciliation 67.

Commission des conflits d'intérêt public 709.

Constitution, 581.

Fonction, 582.

Procédure, 583-596.

Commission des relations du travail 61; 736.

Common law 418-422; 618.

Compérages 455.

\section{Conciliateur} $66 ; 570-571$.

\section{Conciliation} $66-68 ; 502 ; 567 ; 572$.

Procédure, 400.

Volontaire et obligatoire, 570.

Concurrence syndicale (voir rivalité intersyndicale)

\section{Confédération canadienne} 29.

Confédération des syndicats nationaux (CSN)

493; 521 .

Effectifs, 85.

Structure, 85.

Conflit (du travail)

Atténuation des effets des, 567-574.

Intérêt public, 566-646.

Réglementation des formes de, 605-645.

Rôle du, 392-403; 566.

Situation d'urgence, 575-595.

\section{Conflits de juridiction} 522-523.

Congrès du travail du Canada (CTC) $341 ; 493 ; 521$.

Effectifs du, 85.

Structure du, 86; 336-339.

Vis-à-vis la loi, 282.

Conseil canadien des relations du travail Constitution de, 770-772.

Fonction conseil, 742; 769.

Rapport annuel, 773.

Conseil canadien des relations ouvrières $63-64 ; 435 ; 442 ; 448-454 ; 456-457$; $475-476 ; 490 ; 492 ; 495-499 ; 510$; $517 ; 600 ; 703 ; 734-751$.

Détermination des unités de négociation, 444-453.

Implications constitutionnelles, 765-766.

Juridiction, 737-741.

Nouveau nom, 734.

Poursuite pour pratiques déloyales, 476.

Réexamen des décisions, 749-750.

Représentation, 736.

Représentation tripartite, 736.

Requête pour réexamen, 452.

Révocation d'accréditation, 466.

Rôles et attitudes 735 .

Conseil du patronat 351.

Conseil économique du Canada $141-146 ; 156-160 ; 647 ; 671$.

\section{Conseillers juridiques} 92.

\section{Consommation}

Indice des prix à, 653 .

Prix à la, 217.

Constitution des Etats-Unis (1791) 24.

Construction (industrie de la) $459 ; 522 ; 523 ; 531$.

\section{Consultants}

92. 


\section{Convention collective}

69; 194; 196; 198; 202.

Application, 596-604.

Changements en respect avec, 677.

Clauses expérimentales et ajustement, 561-564.

Convention de longue durée, 559-560.

Droit de grève et lockout à l'expiration, 557-569.

Durée, $205 ; 453 ; 559-560$.

Extension, 717.

Formes, distribution et langue, 565 .

Modifications durant la vie, 675 .

Ratification, 500-506.

Sujet à contestation, 455 .

Conversion industrielle 42.

Adaptation des travailleurs, 673-685.

Négociation collective, 383-391.

Notion, 382.

Rôle, 383.

Syndicalisme, 386-388.

Coopération fédérale-provinciale

Législation, 755-756.

Uniformisation des lois; 654; 666; 670; $762 ; 763$.

Cotisations syndicales (retenue des)

Ouvrière, 481-484; 490.

Patronale, 531.

C. P. 1003 58.

Cycle économique 208-209.

Déclaration canadienne des droits (1960) 24.

Déclaration des droits de l'homme (1789) 24.

Déclaration des droits de l'homme (1948) 26.

Déclaration d'indépendance (1776) 24.

Déclaration des droits (1689)

$$
24 .
$$

Délégation de pouvoir $755-756$.

Démocratie économique

Apport fondamental, 300 .

Définition, 296.

Démocratie syndicale 485-499; 509.

Droits des syndiqués (Charte des) 491-493.

Durée du travail 692-694; 713; 718 .

\section{Emploi}

Niveau, 647.

Plein emploi et stabilité des prix, 654

Employés 77.

Exclus des unités de négociation, 436.

Extension des droits de négociation, 437. 443.

Employeurs

(Voir Patronat)

Enquêtes 11-12.

Entrepreneurs dépendants 90.

Entreprises 73-75.

Relevant de deux provinces, 755 .

Etat

Compétence provinciale, 93-94.

Intervention, 33-36; 57; 63; 151; 400; 432; 568.

Législateur, 93-94.

Rôle, 699-773.

Etat-employeur 97; 700-705.

Extension d'une convention collective $70 ; 717$.

\section{Formation} 721-727.

Formule Pearson 232-235.

Freedman (Rapport) 674-676.

\section{Grande Charte (1215)} 24.

Grève

$68 ; 119-120 ; 393 ; 404-411 ; 440-557$; 596-604; 605-610.

Assurance, 532-534.

Droit acquis à l'expiration de la convention collective, 557-569.

Situation d'urgence, 575-595.

Vote, 500-506.

Griefs $107 ; 555$.

Arbitrage, 606.

Conventions collectives à long terme, 559.

Médiation, 599.

Gouvernement (Voir Etat)

Groupements d'employés 80.

Groupements d' "entrepreneurs dépendants 》

90. 
Heures de travail 692-694; 718 .

\section{Immigrants} 46.

\section{Inflation}

$$
\text { 135-136; } 163 .
$$

Augmentation de salaires, 220.

Causes canadiennes, 172-191.

Causes théoriques, 165-171.

Chômage, 652.

Comportement cyclique, 209-210; 647

Indice des prix à la consommation, 653 .

Négociation collective, 218-239.

Rôle du syndicat, 218 .

\section{Information} 721-727.

\section{Injonction} 640-645.

\section{Intérêt public} 98; 488; 502; 539; 557

Commission des conflits d'intérêts public, 581-595.

Conflit du travail, 566-646.

Effets de négociation collective, 646-698.

Situation d'urgence, 575-595.

\section{Législation du travail}

Common law, 418-422.

Loi et comportement des parties, 412 426.

Résistance patronale, 415.

Rôle des lois, 412-413.

Liberté d'association 433-446; 530-534.

Extension, 435.

Liberté d'expression

Patronat, 529.

Piquetage, 613.

\section{Licenciements collectifs} Préavis, 679.

\section{Lock-out}

68; 119; 393; 404-411; 440; 506; 507 ; 532-536; 596-610.

Loi (application de) 636-638.

Loi du travail (voir législation du travail) Arbitrage des chemins de fer (1903), 50.

Code criminel (1900), 49.

Conciliation (1900), 50.

C.P. 1003 (1944), 58.

Décrets de la convention collective du Québec (1934), 70, 717.

Enquêtes en matière de différents industriels (1907), 51-55.

Relations industrielles et sur les enquêtes visant les relations du travail (1948), 59.
Syndicats ouvriers (1872), 49.

Wagner, 56.

\section{Main-d'oeuvre}

76.

Définition, 109.

Déplacement, 679.

Mobilité, 364-375; 697-698; 715.

Politique, 57.

Rémunération, 206-217.

Répartition, 214.

Maîtrise

Droit d'association et négociation, 437.

Maraudage $464 ; 521$.

Marché du travail

Définition et rôle, 101.

Fonction, 363.

Imperfections, 365-366.

Négociation collective, 359; 363-375.

Régime canadien de relations du travail, 430.

Médiation $502 ; 567 ; 599$.

Mémoires (présentation de) 14.

Méthodologie 9-15.

Milieu constitutionnel 47-72.

Milieu économique $38-46$.

Milieu juridique 47-72.

Milieu politique 38-46.

Milieu social 38-46.

«Ministère des relations du travail du Canada 》 706-734.

Ministère fédéral du travail 706-734.

Coordination avec les autres ministères, 732-733.

Relations fédérales-provinciales et internationales, 728-731.

\section{Négociation collective}

Acceptation du principe, 270-283.

Aliénation, 306.

Attitude des employeurs, 272-2.75.

Attitude des syndicats, 276-278.

Bonne foi, 544-547; 570.

But, 63; 362.

Calendrier et durée, 556-558.

Caractéristique, 392. 
Centralisation et décentralisation, 311; 553-555.

Centre de décision, 553-555.

Changements, 123-134.

Changements technologiques, 288 ; 673685.

Clauses expérimentales, 561-564.

Conditions d'emploi, 293-294.

Conversion industrielle, 382-391.

Critique, 250-426.

Déficiences structurelles, 203.

Définition, 106; 431.

Délégation, 756 .

Droit, 437.

Droit des successeurs, 460-461.

Effets, 646-698.

Efficacité, 284-290.

Entre syndicats, 295.

Extension des matières négociables, 285.

Forme, 198-202.

Fragmentation, 40.

Freedman (formule), 674-676.

Groupes exclus, 252-255.

Harmonisation des objectifs, 646-672.

Inflation, 218-239; 647.

Influence, 228-229; 292.

Inquiétude du public, 411 .

Intérêt public, 646-698.

Juridiction fédérale, 754.

Liberté des employés, 433-444.

Liberté des employeurs, 433-446.

Limites, 390-391.

Lockout collectif, 532.

Marché du travail, 359; 363-375.

Mobilité de la main-d'oeuvre, 697-698.

Négociation continue, 561-564.

Notion, 381.

Objectifs, 291.

Participation au progrès de l'entreprise, 695-696.

Patronat, 354.

Portée, 110.

Processus, 107; 544-565.

Productivité, 358.

Protection des droits des travailleurs, 256-258.

Rajustements, 561-564.

Ratification, 500-506.

Reconnaissance, 270-283.

Régime canadien, 567.

Rendement économique, 357-362.

Répartition des revenus, 376-382.

Responsabilité, 279-283.

Rôle, 192-249.

Rôle du conflit, 392-403.

Salaire, 292.

Situation d'urgence, 575-594.

Stratégie, 354.

Structure, 202; 205; 549-552.

Unités (plusieurs), 451.

Valeurs fondamentales, 129.
Négociation de productivité

Effet sur structure de salaire, 696.

Participation aux profits et progrès, 695 .

Normes de travail

114-117; 266-269.

Autorité fédérale, 764 .

Législation, 711-721.

Objectifs nationaux 138-149.

Observations

Recommandations, 427-773.

Organisation syndicale

61

Parité de salaire 236.

\section{Patronat}

Association d'employeurs, 350.

Assurance-grève et lockout, 532-535.

Attitudes et politiques, 538-543.

Changement de nom des employeurs, 460-461.

Droits d'association, 530.

Droits et obligation du, 529-543.

Evolution dans la philosophie, 355.

Gouvernement fédéral comme employeur, 700-705.

Philosophie, 351; 352; 353.

Pratiques déloyales, 467-480; 536-537.

Préavis de changement technologique, 679.

Régime canadien de relations du travail, 430.

Relations du travail, 344-356.

Résistance aux lois, 415.

Services de relations du travail, 349.

Stratégies en relations du travail, 354 .

Syndicalisation, 344-345; 347.

\section{Piquetage}

$417 ; 425 ; 611-645$.

Application de la loi, 636 .

Collectif, 630 .

Délits du travail, 614-619.

Extension des droits, 639.

Juridiction fédérale-provinciale; 762-764.

Légitimité, 623-625.

Notion, 611-613.

Primaire, 627.

Refus de traverser la ligne, 605-627.

Secondaire, 628.

Placement

Bureau, 486-488.

\section{Planification}

126-127; 514 
Plein emploi 155-156; 654

Politique de main-d'oeuvre (Voir main-d'oeuvre)

Politique de prix 181-185.

Politique de revenu 662-664.

Politique fiscale $176 ; 178 ; 655-656$.

Politique monétaire $176 ; 178 ; 655-656$.

Politiques syndicales $425 ; 527-528$.

Poursuites pour pratiques déloyales 476-477.

Pouvoir syndical 485-499.

Pratiques déloyales

Liberté des employeurs, 536; 537.

Protection des syndicats, 467-480.

Présyngob $482 ; 490 ; 516 ; 531$.

Preuve (fardeau de) 639.

Principes de base du régime 22-37.

\section{Prix}

Comportement, 206-207.

Hausse de salaire, 220-222.

Stabilité, $155-156 ; 647 ; 654$.

Plein emploi, 654.

Variation, 157.

Procédure de règlement des griefs 107

Productivité 217.

Indice, 648.

Hausse de salaire, 222.

Salaires réels, 219.

Professions libérales 112.

Droit de négociation, 441.

Public

Torts et désagréments, 404-412.

\section{Ratification}

Vote, 500-506.

Recommandations

Observations, 427-773.

Recherche $10 ; 721-727$.

Reconnaissance

Cessation, 463-467.

Volontaire, 455.
Régime de relations du travail

Acceptation du régime, 428.

Adhésion aux fondements essentiels, 427.

Canada, caractéristiques, 429-430; 481 .

Définition, 21.

Evolution et fonctionnement, 38-122.

Principes de base, 22-37.

Résultats, 118-121.

Rôle du conflit, 392-403.

Règlements des griefs 107.

Relations du travail

Politique, 427-433.

Rôle du gouvernement, 574.

Relations fédérales-provinciales 728-731.

Relations industrielles

Rôle du gouvernement, 574 .

Relations internationales 728-731.

Rencontres $13 ; 15$.

Répartition des revenus (Voir revenus)

Retenue des cotisations (Voir cotisations)

Revenus (répartition des)

Négociation collective, 376-382.

Rivalité intersyndicale $43 ; 263 ; 332-334 ; 519-523$.

Rôle du gouvernement 699.

Disparités, 211; 214

Formule Pearson, 232-235.

Hausse de prix; 220-222.

Minimum, 711-712; 714; 716-717.

Moyenne, 212.

Parité, 236; 687; 691.

Réels et productivité, 219

Statistiques, 649.

Structures, 212; 216-217.

\section{Salaires}

Ajustement, 157.

Baisse, 224-225.

Comportement des parties du revenu national, 206-208; 210; 217.

Contrôle sur, 661-662.

Secteur public

Grève, 409-410.

Sécurité sociale $148-149 ; 286$.

Formule, 320 .

Sécurité syndicale $320 ; 324 ; 481 ; 484 ; 490 ; 494$.

Service de la main-d'oeuvre du Canada 487. 
Sociétés américaines 43-44.

Société de consommation 308-309.

\section{Successeur} 460.

Suède 656

Nombre de grèves, 406.

\section{Syndicalisme} 79-80.

Action politique syndicale, $88-89 ; 513$ 518.

Aliénation, 304-305.

Attitude et politique syndicales, 527-528.

Bilinguisme, 343 .

But, 87.

Concurrence syndicale, 332-334.

Conversion industrielle, 386-388.

Croissance, 81.

De boutique, 257 .

Inflation, 218.

Marché du travail, 363-375.

Mécontentement des travailleurs, 307.

Méthodes du syndicalisme, 331-343.

Mobilité de main-d'oeuvre, 363-375.

Pénétration, 82-83.

Philosophie, 342.

Pouvoir syndical, 485-499.

Raisons d'acceptation, 376.

Réaction du patronat, 344 .

Réformes de structure, 335 .

Régime canadien de relations du travail, 430.

Retenue des cotisations, 481-484.

Rivalité intersyndicale, 332.

Rôle, 89.

Sécurité syndicale, 481-484.

Sortes, 331.

Structure, 84-85; 87; 330-343; 525-526.

Violation de la loi, 423.

Syndicalisme de cadres 437.

Syndicalisme international

Autonomie du syndicalisme canadien, 340.

Structures syndicales, 337-339.

\section{Syndicat de travailleurs}

Accès, 323.

Accréditation, 454-459.

Action politique, 88-89; 513-518.

Boutique, 257.

Changement de nom, 460-461.

Concurrence syndicale, 519-523.

Conflit de juridiction, 522 .

Définition, 111.

Degré de représentativité, 456.

Droits et obligations, 447-528.
Effectifs syndicaux, 485-499.

Expédition au droit de négociation, 463466.

Grève et lockout, 605-610.

Inflation, 218.

Influence sur le salaire des travailleurs, 292.

Juridiction fédérale et provinciale, 757 761.

Objectif fondamental, 119.

Pratiques déloyales, 475.

Ratification et vôte de grève, 500-506.

Régime canadien de relations du travail, 430.

Représentativité, 259.

Responsabilité, 319-330; 507-513.

Responsabilité limitée aux actifs, 511.

Restriction du commerce, 635.

Retenue obligatoire des cotisations, 483.

Statut légal, 508 .

Structure, 84-85.

Taxes d'entrée 490.

Travailleurs 76-78; 485-499.

Aliénation, 301-306.

Droit, 319-330.

Impatience, 307-318; 554.

Mécontentement, 554.

Tribunal

424.

Unité de négociation

$195 ; 204 ; 437 ; 444 ; 447-453 ; 462$; 492.

Critères de détermination, 448.

Employés exclus, 436.

Fusion, 462.

Importance stratégique, 447.

Morcellement, 451.

Unité spéciale pour fins spécifiques, 453.

Urgence (situation de)

Négociation collective, 575-595.

Violence

425.

Vote de grève 505-506.

Vote de ratification 500-504.

Vote de représentation 457. 


\section{SUBJECT INDEX}

\section{Canadian Industrial Relations}

THE TASK FORCE ON LABOUR RELATIONS (T.F.L.R.)

\section{Affluent society 308-309.}

Agency fee $482 ; 490 ; 516 ; 531$.

\section{Alienation}

Causes, 302-303 ; 554.

Collective bargaining, 306.

Unions, 304-305.

Workers, 301-306.

American associations 43-44.

\section{Arbitration} 69

Arbitration award, 603.

Compulsory arbitration, 394-498.

\section{Australia}

Number of strikes, 407.

\section{Bargaining units}

$195 ; 204 ; 437 ; 444 ; 447-453$; $462 ; 492$.

Determination of, 448 .

Division of, 451.

Employees excluded, 436.

Merging of, 462.

Special units for common ends, 453.

Strategic importance, 447.

Bilingualism-Biculturalism

Unionism, 343.

Bill of rights (1689)

24

Boycotting

611-645.

Goal of, 620 .

Jurisdiction of, federal, provincial, 762-764.

Briefs (Submission of) 14

British North America Act $47 ; 52-56 ; 752-769$.
“Canada Department of Industrial Relations" 706-734.

Canada Department of Labour 706-734.

Coordination with other departments, 732-733.

Federal-provincial, and international relations, 728-731.

Canada Labour Relations Board $63-64 ; 435$; 442 ; 448-454 ; 456$457 ; 475-476 ; 490 ; 492 ; 495$. 499 ; $510 ; 517$; 600 ; 703 ; 734751.

Constitutional Implications, 765-766.

Decertification, 466.

Determination of bargaining units, 444453.

Judicial review of decisions, 749-750.

Jurisdiction, 737-741.

New name, 734 .

Prosecution for unfair labour practices, 476.

Representation 736.

Request for review of decisions, 462.

Roles and attitudes, 735.

Structure, tripartite, 736.

Canada Manpower Service 487

Canadian Labour (Standards) Code 713

Canadian Bill of Rights (1960) 24

Canadian Chamber of Commerce $91 ; 271 ; 279$.

Canadian Construction Association $91 ; 531$.

Canadian Confederation 29

Canadian Industrial Relations Board 734-751.

* This index was prepared by Jean Sexton to facilitate consultation of the Woods Report. The numbers following the entries refer to the paragraphs. 


\section{Canadian Industrial Relations Council}

Annual Report, 773.

Formation, 770-772.

Function, $742 ; 769$.

Canadian Labour Congress (CLC) $341 ; 493 ; 521$.

Law, and the, 282.

Strength of, 85 .

Structure of, $86 ; 336-339$.

Canadian Manufacturer's Association $91 ; 272$.

\section{Canadian Pulp and Paper Association} 91.

\section{Capitalism} $30 ; 32 ; 273$.

\section{Carter Commission 160.}

\section{Centralization}

In the collective bargaining process, 553555.

\section{Centre des dirigeants d'entreprise 91}

\section{Certification}

$61 ; 63 ; 64 ; 259-265 ; 394$

Decertification, termination, $463 ; 465$; 466.

Duration, 464.

Representation votes, 454 .

Temporary certification (construction) 459.

Unions, 454-459.

Voluntary recognition, 454 .

\section{Check-off}

(see union dues)

\section{Closed shop}

$$
324 .
$$

Definition, 320.

\section{Collective action} $433-446$; 530-534.

\section{Collective agreement} $69 ; 194 ; 196 ; 198 ; 202$.

Application of, 596-604.

Challenges to, 455.

Changes regarding, 677.

Duration of, $205 ; 453$; 559-560.

Experimental clauses and adjustments, 561-564.

Extension, 717.

Form, Distribution, and Language of, 565.
Long term contracts, 559-560.

Modifications during life of, 675 .

Ratification of, 500-506.

Right to strike and lockout on termination of, 557-569.

\section{Collective Bargaining}

Acceptance in principle, 270-283.

Agreement adjustments, 561-564.

Alienation, 306.

Attitude of employers, 272-275.

Attitude of unions, 276-278.

Bargaining units, 451.

Basic values, 129.

Between unions, 295.

Canadian system, 567.

Centralization and decentralization, 311; 553-555.

Changes in, 123-134.

Characteristics, 392.

Collective lockout, 532.

Conditions of employment, 293-294.

Conflict, role of, 392-403.

Continuous bargaining, 561-564.

Coverage of, 110.

Critique of, $250-426$.

Definition, 106 ; 431.

Delegation of power, 756 .

Distribution of income, 376-382.

Economic efficiency, 357-362.

Effectiveness, 284-290.

Effects, 646-698.

Emergency situation, 575-594.

Expansion of negotiable matters, 285.

Experimental clauses, 561-564.

Federal jurisdiction, 754 .

Forms, 198-202.

Fragmentation, 40.

Freedman formula, 674-676.

Freedom of employers, 433-446.

Freedom of workers, 433-444.

Goals, 63 ; 362.

Good faith, 544-547; 570.

Groups excluded from, 252-255.

Harmonization of goals, 646-672.

Idea of, 381.

Industrial conversion, 382-391.

Inflation, 218-239; 647 .

Influence, 228-229; 292.

Labour market, 359 ; 363-375.

Labour mobility, 697-698.

Law, 437.

Limitations, 390-391.

Locus of decision making, 553-555.

Management, 354.

Objectives, 291.

Participation and profit sharing, 695-696.

Process, 107 ; 544-565.

Productivity, 358. 
Protection of workers' rights, 256-258.

Public interest, 646-698.

Public restiveness, 411 .

Ratification, 500-506.

Recognition, 270-283.

Responsibility, 279-283.

Schedule and duration, 556-558.

Strategies, 354.

Structural deficiencies, 203.

Structure, $202 ; 205 ; 549-552$.

Successor rights, 460-461.

Technological change, $288 ; 673-685$.

Wages, 292.

Common law $418-422 ; 618$.

\section{Conciliation} $66-68 ; 502 ; 567 ; 572$.

Procedures, 400.

Voluntary and compulsory, 570.

\section{Conciliation officer} $66 ; 570-571$.

Confederation of National Trade Unions (CNTU) $493 ; 521$.

Stregth, 85.

Structure, 85

Conseil du patronat 351.

Constitutional environment 47-72.

Construction industry $459 ; 522 ; 523 ; 531$.

Consultants (Experts) 92.

Consumption

Corisumer Price Index, 653.

Consumer prices, 217.

\section{Courts} 424.

Criminal Code $49 ; 256 ; 442$.

Breach of contract, 632 .

Restraint of trade, 635.

Unfair labour practices, 634 .

Union activity, 631.

"Watching and besetting", 633.

Decision making, Locus of

Collective bargaining, 553-555.

Declaration of Human Rights (1948) 26.

Declaration of Independence (1776) 24.

Declaration of the Rights of Man (1789) 24
Delegation of power 755-756.

"Dependent contractor" organizations 90.

Displacement Notice, 679.

Distribution of income (see income)

Economic Council of Canada $141-146 ; 156-160 ; 647 ; 671$.

Economic cycle 208-209.

Economic environment $38-46$.

Economic planning $126-127 ; 514$.

Emergency situation

Collective bargaining, 575-595.

Employee organizations 80.

Employees 77.

Excluded from bargaining units, 436 .

Extension of the rights of collective bargaining, 437-443.

Employers (see management)

\section{Employer associations} $62 ; 91$.

Certification, 531.

Collective bargaining, 350 .

Freedom of association and collective action, 530-534.

Wider employer alliances, 535.

Employment

Full employment and price stability, 654.

Level of, 647.

Enterprises 73-75.

Inter-provincial, 755 .

Extension of collective agreements $70 ; 717$.

Federal-provincial cooperation

Legislation, 755-756.

Standardization of laws, $645 ; 666 ; 670$ : $762 ; 763$.

Federal-provincial relations 728-731. 
Fiscal policy $176 ; 178 ; 655-656$.

Freedman Report 674-676.

Freedom of association $433-446$; $530-534$.

Freedom of speech

Management, 529.

Picketting, 613.

French Canada 45.

Full employment $155-156 ; 654$.

\section{Good faith}

Collective bargaining, 544-548.

Government (see State)

Grievances $107 ; 555$.

Arbitration, 606.

Long term contracts, 559.

Mediation, 599.

Grievance procedures 107.

Hours of work $692-694 ; 713 ; 718$.

Immigrants 46.

Incomes and Costs Research Board Federal-provincial cooperation, 767. Terms of reference, 666-672.

\section{Income (distribution of)}

Collective bargaining, 376-382.

Incomes policy 662-664.

Industrial Conflict

Emergency situations, 575-595.

Mitigating the effects of, 567-574.

Public interest, and, 566-646.

Regulating the forms of, 605-645.

Role of, $392-403$; 566.

\section{Industrial conversion} 42

Collective bargaining, 383-391.

Definition and description of, 382 .

Human adjustment to, 673-685.

Role of, 383.

Unions and, 386-388.
Industrial democracy

Basic contribution of, 300 .

Definition of, 296.

Industrial inquiry commission 574.

Industrial relations

Role of government, 574.

Industrial relations system

Acceptance of foundations, 427.

Canada, characteristics, $429-430 ; 481$.

Conflict, role of, 392-403.

Definition of, 21.

Endorse basic principles, 428.

Evolution and working of, 38-122.

Fundamental principles, 22-37.

Results, 118-121.

Inflation

$135-136 ; 163$.

Canadian causes of, 172-191.

Collective bargaining, 218-239.

Consumer Price Index, 653.

Cyclical behaviour, 209-210; 647 .

Role of Unions, 218.

Theoretical causes of, 165-171.

Unemployment and, 652 .

Wage increases and, 220.

Information 721-727.

Initiation fees 490.

Injunction 640-645.

International relations 728-731.

International unionism

Canadian autonomy of unions, 340 .

Union structures, 337-339.

Inter-union rivalry $43 ; 263 ; 332-334 ; 519-523$.

Investigations 11-12.

Jurisdiction or work assignment disputes 522-523.

Labour force

Allocation, 214.

Definition, 109.

Displacement, 679.

Early policies, 57.

Mobility, 364-375; 697-698 ; 715.

Remuneration, 206-217. 


\section{Labour legislation}

Collective Agreement Decrees Act of Quebec (1934), $70 ; 717$.

Common Law, 418-422.

Conciliation (1900), 50.

Criminal Code (1900), 49.

Employer resistance, 415.

First federal labour legislation (1872), 49.

Industrial Disputes Investigation Act (1907), 51-55.

Industrial Relations and Disputes Investigation Act (1948), 59.

Law and the behaviour of the parties, 412-426.

Order in Council, P.C. 1003 (1944), 58.

Railway Labour Disputes Act (1903), 50.

Role of laws, 412-413.

Wagner Act (1935), 56.

\section{Labour market}

Canadian industrial relations system, 430.

Collective bargaining, 359 ; 363-375.

Definition and role, 101 .

Function, 363.

Imperfections in, 365-366.

\section{Labour relations}

Politics of, 427-433.

Role of government, 574.

Labour relations boards

$$
61 ; 736 \text {. }
$$

Labour standards

$$
114-117 \text {; 266-269. }
$$

Federal jurisdiction, 764.

Legislation, 711-721.

Law (application of) 636-638.

Law enforcement officers

Right of association, 439-440.

Legal counsel and consultants 92.

Legal environment 47-72.

\section{Liberal professions} 112

Bargaining rights, 441.

Lock-out

$68 ; 119 ; 393 ; 404-411 ; 440 ; 506$; $507 ; 532-536 ; 596-610$.

\section{Magna Carta (1215)} 24.

Managerial employees supervisory and junior

Right of association and negotiation, 437.
Managerial employees, unionism of 437.

Mediation

$502 ; 567 ; 599$.

Meetings

$13 ; 15$.

Methodology 9-15.

Monetary policy

$176 ; 178 ; 655-656$

\section{National objectives}

138-149.

\section{Observations}

Recommendations, 427-773.

\section{Open union shop}

Definition, 320.

Order in Council, P.C. 1003 58.

\section{Pearson Formula} 232-235.

\section{Personnel administration}

Definition, 104.

\section{Picketing}

Application of the law of, 636 .

Collective, 630 .

Extension of the rights of, 639.

Federal-provincial jurisdiction, 762-764.

Idea of, 611-613.

Industrial torts, 614-619.

Legality, 623-625.

Primary, 627.

Secondary, 628.

Refusal to cross picket line, 605-627.

\section{Placement Bureau} 486-488.

Political activities of unions 88-89; 513-518.

\section{Political environment} 38-46.

\section{Prices}

Behaviour, 206-207.

Full employment, 654.

Stability, $155-156 ; 647 ; 654$.

Variation, 157.

Wage increases, 220-222.

Pricing policies 181-185.

Principles underlying the system 22-37. 
Productivity

217

Index, 648.

Real wages, 219.

Wage increases, 222.

\section{Productivity bargaining}

Effects on wage structure, 696.

Progress and profit sharing, 695.

\section{Proof (burden of)} 639

Prosecution for unfair labour practices 476-477.

Public

Inconvenience and hardship, 404-412.

Public interest

$$
98 ; 488 ; 502 ; 539 ; 557 .
$$

Effects of collective bargaining, 646-698.

Emergency situations, 575-595.

Industrial conflict, 566-646.

Public Interest Disputes Commission, 581-595.

Public Interest Disputes Commission 709.

Formation, 581.

Function, 582 .

Procedures, 583-596.

Public sector

Strikes, 409-410.

Raiding between rival unions $464 ; 521$.

Railway Association of Canada 91.

Ratification vote 500-506.

Recognition

Termination, 463-467.

Voluntary, 455.

Recognition of associations 454-455.

Recommendations

Observations, 427-773.

Representation vote 457.

Research

$10 ; 721-727$.

Rights of union members 491-493.

Role of government 699.
Social environment 38-46.

Social security $148-149 ; 286$.

Formula, 320.

State

Intervention, $33-36 ; 57 ; 63 ; 151 ; 400$; $432 ; 568$.

Legislator, 93.

Provincial jurisdiction, 93-94.

Role of, 699-773.

State as employer $97 ; 700-705$.

Strike insurance 532-534.

Strikes

68 ; $119-120 ; 393$; 404-411 ; 440557 ; 596-604; 605-610.

Insurance, 532-534.

Potential emergency situation, 575-595.

Right acquired on the expiration of a collective agreement, 557-569.

Vote, 500-506.

Strike vote 505-506.

Successor rights 460.

Sweden

656

Number of strikes, 406.

"Sweetheart" agreements 455

Technological change (see industrial conversion) $288 ; 673-685$.

and workers' discontent, 310 .

Trade union

Access to, 323.

Basic goals, 119.

Canadian industrial relations system, 430.

Certification, 454-459.

Change of name, 460-461.

Company union, 257.

Compulsory check-off, 483.

Conflict of jurisdiction, 522 .

Cooperation among unions, 519-523.

Definition, 111.

Federal-provincial jurisdiction, 757-761.

Inflation, 218.

Influence on wages and salaries, 292.

Legal status, 508.

Limited (corporate) liability, 511. 
Political action, 88-89; 513-518.

Ratification and strike votes, 500-506.

Representation, 259.

Responsibility, 319-330; 507-513.

Restraint of trade, 635 .

Rights and obligations, 447-528.

Strikes and lock-outs, 605-610.

Structure, 84-85.

Termination of bargaining rights, 463466.

Unfair labour practices, 475.

Worker support, 456.

\section{Trade unionism}

Alienation, 304-305.

Bilingualism, 343.

Canadian industrial relations system, 430.

Company unions, 257.

Cooperation among unions, 332-334.

Goals, 87.

Growth, 81.

Industrial conversion, 386-388.

Inflation, 218.

Inter-union rivalry, 332 .

Labour market, 363-375.

Labour mobility, 363-375.

Management reaction to, 344 .

Philosophy of, 342 .

Political activities of unions, 88-89 ; 513-518.

Rationale for, 376.

Role of, 89.

Structure, $84-85$; 87 ; 330-343 ; 525526.

Structural reforms, 335 .

Types of, 331.

Union attitudes and policies, 527-528.

Union authority, 485-499.

Union dues and the check-off, 481-484.

Union methods, 331-343.

Union penetration, 82-83.

Union security, 481-484.

Violations of the law, 423.

Worker discontent, 307.

\section{Unemployment}

Cost of, 162.

Cyclical, 692-694.

Inflation, and, 652 .

Insurance, 56.

Rate of, 159.

\section{Unemployment insurance 56.}

\section{Unfair labour practices}

Freedom of employers, 536; 537.

Protection of unions, 467-480.
Union affiliation

Changing of, $458 ; 465$.

Union authority 485-499.

Union democracy 485-499; 509.

Union dues (deduction of)

Employer, 531.

Worker, and, 481-484; 490.

Union organization

61.

Union policies and approaches $425 ; 527-528$.

Union security $320 ; 324 ; 481 ; 484 ; 490 ; 494$.

Union shop

Definition, 320.

United Nations Charter 26.

United States Constitution (1791) 24.

Violence

425.

Wage parity

236.

Wages

Adjustments, 157.

Averages, 212.

Behaviour on national income, 206-208;

$210 ; 217$.

Control, 661-662.

Disparities, 211 ; 214.

Downward movement, 224-225.

Minimum, 711-712; 714; 716-717.

Parity, $236 ; 687$; 691 .

Pearson formula, 232-235.

Price increases, 220-222.

Real, and productivity, 219.

Statistics, 649.

Structures, 212 ; 216-217.

Workers

$76-78 ; 485-499$.

Alienation, 301-306.

Discontent, 554.

Rights, 319-330.

Unrest, $307-318$; 554. 